\title{
PACKAGING DESIGN AND TESTING BY EYE TRACKING
}

\author{
Gregor Franken \\ University of Ljubljana, Faculty of Natural Sciences and Engineering, Department of Textiles, \\ Graphic Arts and Design Ljubljana, Chair of Information and Graphic Arts Technology, \\ Ljubljana, Slovenia
}

\begin{abstract}
The importance of packaging design has been increasing in today's competitive world. Approximately $70 \%$ of purchasing decisions are made in a store. Over $60 \%$ of purchasing decisions are based on packaging; the actual shopping is thus the final chance for the packaging to attract the buyer. Packaging has between 2 and 3 seconds to convince the buyer. In addition to the appearance of the individual packages, the appearance of brand packaging is important. We compared different designs of packages. Finally, we placed individual packages on the shelves in a store and carried out measurement of in-store noting. The measurements were carried out using eye tracking equipment (Tobii X120). For each participant, the observing time and the number of fixations in individual areas of interest were measured; both were then compared with heat maps. In this way, we compared the suitability of the form of individual packages and the salience of the packages on the shelves for potential buyers.
\end{abstract}

Keywords: packaging, design, online observing, fixations, observing time, heat maps

\section{INTRODUCTION}

With steep competition of products on the market, packaging is one of the most important factors in product sale. Design and the search for the optimal design preferred by the buyers, which enhances the recognition on the market, present the greatest challenge for both the designers and the company itself. We might say that packaging is the medium between the buyer and the product, representing the first contact with the consumer. Many purchases are spontaneous, and because of the growing competition on the market, packaging plays a special role in convincing the buyer. From the consumer perspective, packaging also plays a major role when products are purchased: packaging is crucial, given that it is the first thing that the public sees before making the final decision to buy. (Giovannetti, 1995)

In our study, we attempt to determine how the consumer views individual packages, and how he or she reacts to all the packaging on the shelves. Consumer behaviour was tracked using eye-tracking equipment. What is particularly interesting about eye-tracking measurements and testing is the fact that the answers regarding the attention may be completely different from the actual visual attention. Without suitable eye-tracking analyses, any comparison of the design elements of packaging is irrelevant in this day and age. The technology takes into account the physiological movements of the human eye, analysing the psychological consequences of these movements; moreover, accurate judgements or recommendations on how to improve packaging design are based on the data obtained through testing and analyses.

\subsection{Packaging}

The process of packaging can be defined as the activity of designing, as well as the packaging or wrapping of a product. Packaging is much more than just protection of the content; it is an active means of communication on the shelf, generally in the company of other, similar products, representing a contact between potential consumers in the process of purchasing decision making. It is at the same time an advertising medium and a mute salesperson that has less than two seconds to convince the buyer. (Stephenson, 2020)

Packaging decisions have an important impact on the product, as packaging can contribute to maintaining a successful market position of a product, enhancing its positioning, improving the quality of the content when newer materials are used (above all in food sales), reaching new target groups, etc. The importance of packaging is growing for several reasons. One of them is the increasingly stronger competition, which means that the product needs to stand out among all the other similar products. In addition to the design of the individual package, two issues addressed by visual merchandising, i.e., brand packaging design and the salience of packaging on the shelves in a store, are also important. (Morgan, 2016) 
Reiner in Rose (2002) list three points which distinguish advertising from packaging. 1) Packaging without any subconscious influence of advertising represents 70\% of impulsive shopping. 2) Packaging advertises a product before and after purchase. 3) Packaging outlasts an advertisement. (Reiner and Rose, 2020) The next, equally important reason is that the differences in quality and price are decreasing. Products are becoming very similar, and, as a consequence, it is the packaging that attracts the consumer. Moreover, consumers are becoming less loyal, and any detail of packaging, however minor, may attract them. Around $70 \%$ of purchasing decisions are made in the store. (Clark, 2020)

\section{2 Eye-tracking}

Eye-tracking is the process of recording the point of gaze and eye movements in an individual, using equipment relying on optical methods of tracking eye movements. In eye-tracking, infrared diodes are used to create reflection patterns on the cornea. These reflection patterns are collected by video-based sensors along with other visual information on the subject of testing.

\subsubsection{Gaze plots}

Gaze plots show the location, order, and time spent looking at locations on the stimulus, whether packaging, web page, print advertisement, or moving pictures. So the primary function of the gaze plot is to reveal the time sequence of looking or where we look and when we look there. Time spent looking, most commonly expressed as fixation duration, is shown by the diameter of the fixation circles. The longer the look, the larger the circle. (Tobi Pro, 2020)

\subsubsection{Saccades}

When we read or observe, the eyes move in a discontinuous manner. The motion of the eye from one fixation to the next occurs in a rapid jump called the saccade. Saccades are exceptionally rapid eye movements in which we momentarily change the viewing direction and align the image of the observed object with the area of the yellow spot. Saccades are the most frequent eye motion. Their angular velocity can exceed $500^{\circ}$ s. (Hochberg, 1978)

\subsubsection{Fixations}

Fixations are short pauses at picture or at individual text elements during the reading or observing process, and are needed for the brain to process the text being read (Figure 1). Reading fixations typically last from 200 to 250 milliseconds (ms) and are significantly longer when reading unknown or less familiar words (Rayner et al, 2010). In observing elements and pictures, fixations typically last from a few milliseconds to several seconds.

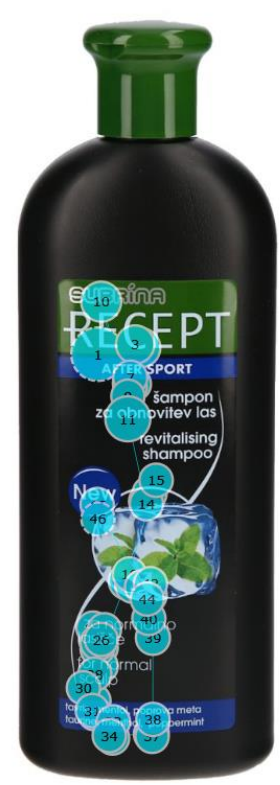

Figure 1: Fixations of one participant detected and recorded with eye-tracker on packaging 


\subsubsection{Heat maps}

A heat map is a graphical representation of how looking is distributed over the stimulus. Heat maps are generally used by online marketers to show what percentage of people interacted with different parts of a web page. This is usually done using software such as eye tracking, click tracking, mouse tracking, etc. Unlike gaze plot, there is no information about the order of looking in a static heat map (Figure 2).

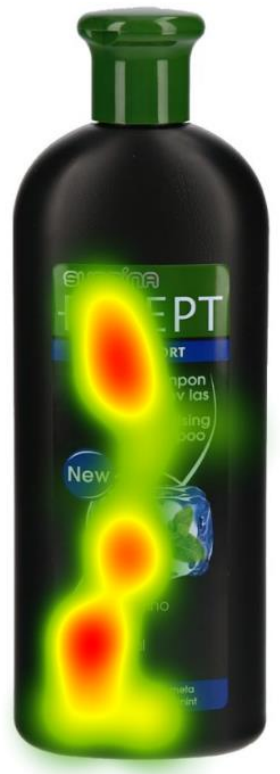

Figure 2: Heat maps detected and recorded with eye-tracker on packaging

\subsection{Form, colour, typography and image}

In designing a new package, the form is certainly of utmost importance. The form is limited by the volume, as it must adjust to the content. The design of the form and it being pleasant to the touch are vital: Will the edges be sharp or soft? Ergonomics is foregrounded here: How will the package feel in our hands? Since we used shampoo as the focus of our packaging design, it is essential to take into account the stability of the package. Will it be prone to toppling over, can it stand upside down on the part which opens... The surface structure of the packaging is crucial. Will it be glossy or soft touch matte? When designing shampoo packaging, the fact that it will mostly be touched with wet hands should be taken into consideration.

The colour is certainly one of the most important factors in packaging. White, blue and yellow dominate in shampoo design. In the context of colour, Fructis Garnier shampoos are worthy of attention: The packaging of these shampoos has stood out with its non-traditional choice of attractive and vibrating apple green. With a suitable advertising campaign and a decisive colour, these shampoo bottles certainly stand out in the colour palette of the other shampoos on the shelf. (Sleever International, 2020)

The typography, the colour palette, the noticeable visual images and the identity of the brand work together to create a synergetic effect, and may eventually end up becoming iconic if rigorously maintained. That is why the choice of typography is so important. In packaging design it is not necessary to use imagery as a design element. A suitably attractive packaging can also be designed without using imagery.

\section{METHODS}

\subsection{Preliminary measurements}

In the preliminary measurements, we chose between two forms of packaging shown on the screen. All participants had normal or corrected-to-normal vision. The study was performed in accordance with the latest declaration of Helsinki. The measurements were performed in a quiet room with walls painted in neutral matte gray, according to the ISO 3664 standard (ISO, 2009). The picture of packaging were displayed on a 24-inch LCD screen with a resolution of $1900 \times 1200$ pixels (pixel size $0.27 \mathrm{~mm}$ ) at a $60 \mathrm{~Hz}$ 
refresh rate in dark characters on light backgrounds. The tested individuals were positioned $60( \pm 1) \mathrm{cm}$ from the screen according to the recommendations of the ISO 9241 standard (ISO, 2011).

\subsection{Main measurements}

In the main study, we used one form packaging with suitable typographic and graphic elements in the preliminary measurements. We limited the observing time for the packaging on the screen to 5 seconds. We marked different target areas of interest (AOI), dividing them into brand, product name, body text and image. For each participant, the number of fixations, fixation duration, the time to first fixation and the total fixation duration in AOI (Figure 3) were measured. Using the method of the longest fixation duration or the so called heat maps, recommendations for changing the graphic elements of the packaging were made. In placing the package on the shelves in the store, the noticeability of the package among other products was measured; the showing time was limited to 3 seconds. On the shelves, AOI was only marked in the spot where our package was placed. In the AOI marked, the number of fixations, the time to first fixation and the whole fixation duration were measured.

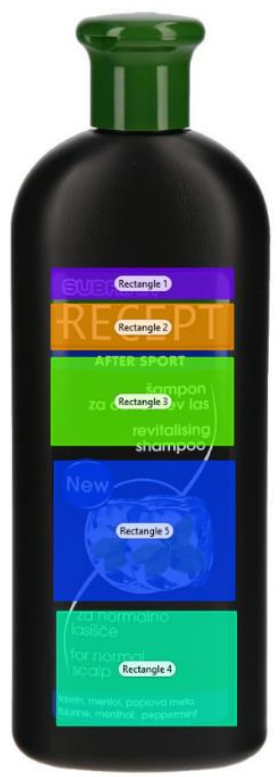

Figure 3: Target area of interest (AOI) on packaging

There were 24 participants, 12 were male and 12 female, ages ranged from 18 to 40, with an average of 23.50 years; all participants had normal or corrected-to-normal vision.

\subsection{Apparatus}

To track the eye movement we used the Tobii X120 eye tracking device and the Tobii Studio 3.1.3 software. The eye tracker tracks the movement of the eye by following the reflection of the image from the cornea. This reflection is generated when the infrared illuminators at the front side of the eye tracker create patterns of light reflecting from the cornea. Eye tracker contains an infrared-sensitive camera that tracks the individual's eye movements and fixations. Prior to the measurement, each individual adapted to the lighting conditions of the room for 5 minutes and then underwent a 9-point screen-based calibration. We also used in shop Tobii Pro Glasses 3 with full HD resolution scene camera with $106^{\circ}$ field of view and Tobii Pro Lab software.

\section{RESULTS}

It was necessary to establish the impact of the different elements of packaging on information reception. Which factors influence changes in eye movement. The time to first fixation, the number of fixations and the fixation duration were compared.

For packaging, the results were very much in accordance with the expectations. The paths of the participants were more or less homogeneous, and their eye movement basically followed the standard 
reading pattern. The time of the first fixation is supposed to be as short as possible, as the participant must focus on the elements of packaging. The participants first focused on textual elements, such as the product name and later on the brand name. This can be attributed to larger font size and the use boldface for the product name. More time was spent on the picture than on the body text. All the data on eye movements and the number of eye movements in packaging observation are given in Table 1.

Table 1: Eye tracking results for packaging design (average of all individual results) within the area of interest (AOI). (1) Time of first fixation. The time of first fixation (in seconds) indicates, when a certain was first focused. (2) Fixation duration. Fixation duration is the average time for fixations. (3) Number of fixations. The fixation count is the amount of fixation. (4) Total fixation duration.

\begin{tabular}{|l|c|c|c|c|}
\hline Gaze plots & (1) & (2) & (3) & (4) \\
\hline Brand & 0.20 & 0.09 & 2.4 & 0.32 \\
\hline Product name & 0.61 & 0.15 & 6.4 & 0.94 \\
\hline Body text & 1.66 & 0.10 & 8.4 & 1.14 \\
\hline Image & 3.84 & 0.05 & 9.1 & 0.42 \\
\hline
\end{tabular}

\subsection{Brand}

The participants noticed the brand of the package the fastest (Figure 4). On average, they needed 0.2 seconds to notice it. The average time of an individual fixation was 0.09 seconds. The number of all fixations in the area of the brand logo was 2.4. The total fixation duration was 0.32 seconds.

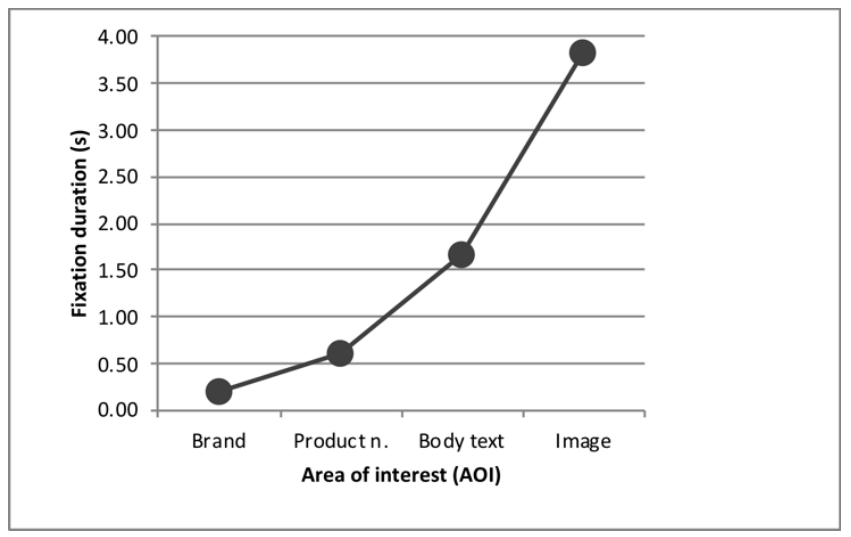

Figure 4: Time to first fixation

\subsection{Product name}

The time of the first fixation was 0.61 seconds on average; the average fixation duration was 0.15 seconds (Figure 5). The total number of fixations on the product name was 6.4. On average, the total fixation duration on the product name was 0.94 seconds.

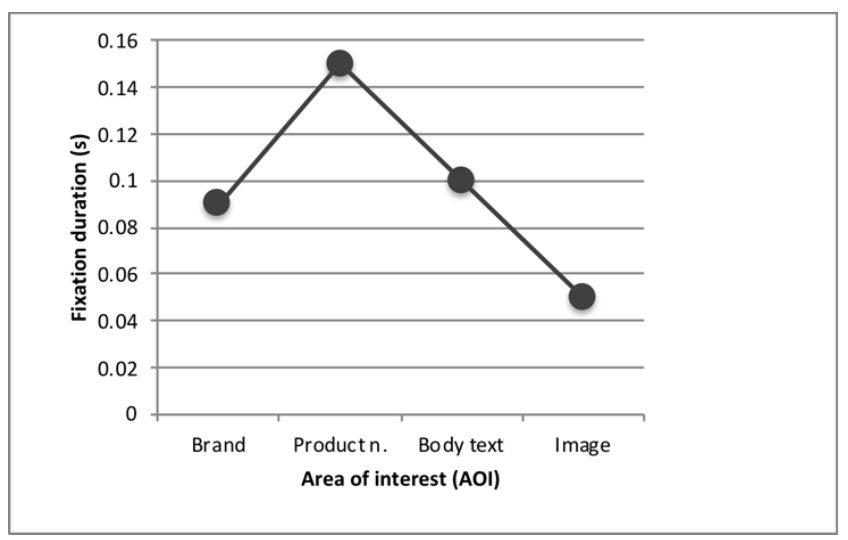

Figure 5: Fixation duration 


\subsection{Body text}

For the body text, the average time of the first fixation was 1.66 seconds and the participants spent an average of 0.1 seconds on it. As body text involves reading, the participants made 8.4 fixations. The total fixation duration was 1.14 seconds (Figure 6).

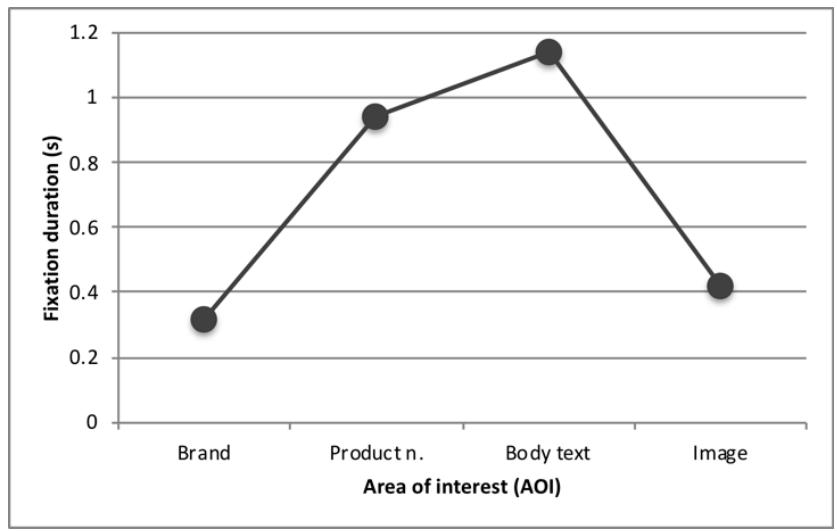

Figure 6: Total fixation duration

\subsection{Image}

The time of the first fixation was 3.84 seconds on average (Figure 7), and an average of 0.15 seconds were spent on the image. The number of fixations was greatest for the image, 9.1; however, the total fixation duration was only 0.42 seconds.

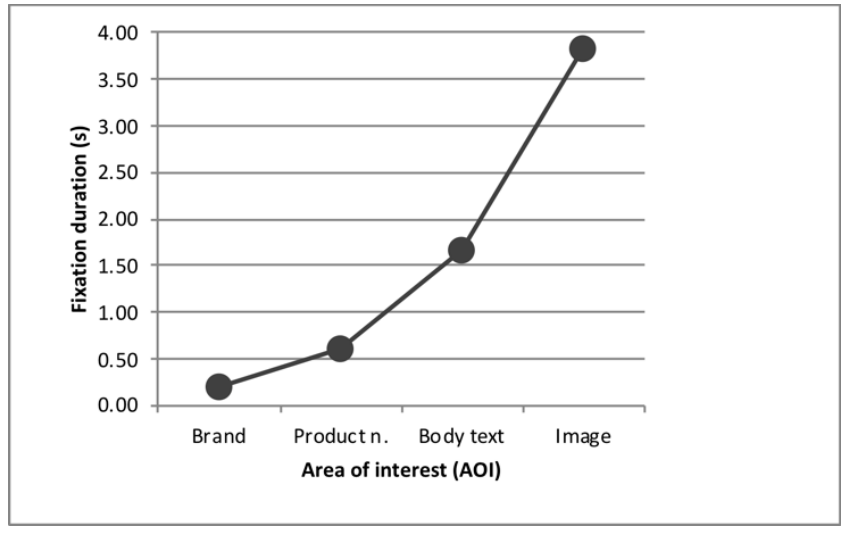

Figure 7: Time of the first fixation

\subsection{Attention to packaging on the sales shelf}

As mentioned above, the participants only had 3 seconds to find the shampoo in question on the shelf in the store. In measuring the fixations, the following results were obtained: the average time to first fixation was 0.67 seconds, the average fixation duration was 0.20 seconds and an average of 6.2 fixations were made on the packaging.

\section{DISCUSSION}

Our results yield four conclusions. First, the form of the packaging: softer forms seem to be preferred. Second, the noticeability of the packaging is influenced by the label itself or the brand logo. The label certainly has the power to attract the consumer, which corresponds to the findings in advertising research (Wedel and Pieters, 2008). Our results suggest that sans serif fonts are more attractive than serif fonts. Similar conclusions have been reported by Ampuero and Vila (Ampuero and Vila, 2006). The right choice of sans serif fonts is also crucial. The strokes of the fonts must be soft and rounded. Third, in packaging design, we used a small image that did not exceed the message value of the colour of 
packaging, the colour of the label and the text on the packaging. Fourth, the results of our study show that the time needed to notice individual packages very much depends on the colour and thus impacts the noticeability among other products on the shelf. It is very important that the colour of the product stands out among other products. In our case, there were several black shampoos on the shelves. Black itself does not stand out, but it creates the feeling of higher price range.

\section{CONCLUSIONS}

The eye tracking method has a number of advantages in the context of marketing research. Its greatest advantage is that it collects data on consumer behaviour directly, thus solving the traditional problem of the discrepancies between what an individual says and what an individual does. Data gathered in this way are more reliable. At the same time, eye movements are direct indicators of an individual's attention, which is difficult to control consciously; as a results, eye tracking resolves the problem of subjective data gathered using interviews or individual focus groups. In marketing research, the eye tracking method means progress in the field of objective data gathering. In terms of marketing, it is worth mentioning that suitable packaging is certainly a key element of marketing itself. The concept of the packaging, its visual elements, its colour and its fonts are crucial in positioning the packaging in strong competition; packaging thus represents an element of marketing. By using advance testing, choosing wrong design elements, which may lead to additional costs associated with redesign, can be avoided; and, above all, potential consumer response can be researched in advance, prior to placing the packaging on the market. It is important to bear in mind that in today's competitive market only some of the new products will make it. In future research on product salience on the shelf, it may be worth comparing the product positioned in different parts of the shelf, as we know that different locations of the product on the shelf play a role in how quickly the product is found; another factor is also the number of other product on the shelf.

\section{REFERENCES}

[1] Ampuero, O., Vila, N.: "Consumer perceptions of product packaging", Journal of Consumer Marketing 23 (2), 100-112, 2006. doi: 10.1108/07363760610655032.

[2] Clark N.: "70\% of purchasing decisions are made in-store", URL: https://economictimes.indiatimes.com/70-of-purchasing-decisions-are-made-instore/articleshow/3816087.cms?from=mdr (last request: 2020-08-10).

[3] Giovannetti, V. M. D.: "El mundo del envase: Manual para el diseño y producción de envases y embalajes", (Editorial Gustavo Gili, México, 1995.).

[4] Hochberg, J.: "Perception (Prentice-Hall foundations of modern psychology series)", (Prentice-Hall, New Jersey, 1978.).

[5] International Organization for Standardization, ISO 3664:2009 Graphic technology and photography - Viewing conditions, International Organization for Standardization, 2009.

[6] International Organization for Standardization, ISO 9241-303:2011, Ergonomics of human-system interaction - Part 303: Requirements for electronic visual displays, International Organization for Standardization, 2011.

[7] Morgan, T.: "Visual Merchandising", (Laurence King Publishing, London, 2016.).

[8] Rayner, K., Timothy, J., Slattery, T. J., Bélanger, N. N.: "Eye movements, the perceptual span, and reading speed", Psychonomic Bulletin and Review 17, 834-839, 2010. doi: 10.3758/PBR.17.6.834.

[9] Reiner T., Rose D.: "Packaging design VS. conventional advertising, Austropack", URL: http://www.berndtundpartner.de/images/pdf/veroeffentlichungen/2002/article_designvsadvertising.pdf (last request: 2020-08-08).

[10] Sleever International: "Fructis - a green shelf revolution", URL: http://www.sleever.com/article/fructis-a-green-shelf-revolution (last request: 2020-08-05).

[11] Stephenson A.: "One, two seconds ... the time your package has to engage your shopper", URL: https://explorerresearch.com/optimize-your-package/ (last request: 2020-07-10).

[12] Tobi Pro: "Working with Heat Maps and Gaze Plots", URL: https://www.tobiipro.com/learn-andsupport/learn/steps-in-an-eye-tracking-study/interpret/working-with-heat-maps-and-gaze-plots/ (last request: 2020-08-08).

[13] Wedel, M., Pieters, R.: "Eye Tracking for Visual Marketing", Foundations and Trends in Marketing 1 (4), 231-320, 2008. doi: 10.1561/1700000011. 


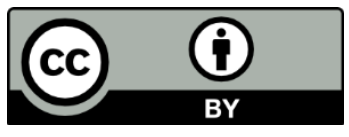

(C) 2020 Authors. Published by the University of Novi Sad, Faculty of Technical Sciences, Department of Graphic Engineering and Design. This article is an open access article distributed under the terms and conditions of the Creative Commons Attribution license 3.0 Serbia (http://creativecommons.org/licenses/by/3.0/rs/). 